\title{
ROLE OF NANOG IN GLIOMA MALIGNANCY DEVELOPMENT AND POTENTIAL AS THERAPEUTIC TARGET
}

\author{
Fergie Runtu ${ }^{1}$ and Novi Silvia Hardiany ${ }^{2 *}$ \\ ${ }^{1}$ Undergraduate Program in Medical Sciences, Faculty of Medicine, Universitas Indonesia, Jakarta \\ Pusat, Indonesia, ${ }^{2}$ Department of Biochemistry and Molecular Biology, Faculty of Medicine, Universitas \\ Indonesia, Jakarta Pusat, Indonesia
}

Recent discoveries have indicated that tumorigenesis arises from a population of cells with self-renewability and pluripotency characteristics. These led to the theory of cancer stem cells, in which these cells carry molecular signatures similar to embryonic stem cells. Homeobox protein NANOG is a transcription factor that helps embryonic stem cells maintain pluripotency. It has been identified to be highly expressed in several types of tumors. Further studies have shown the linkage of NANOG overexpression to malignancy and therapeutic resistance, however very limited research has clearly identified NANOG role in malignancy and therapeutic response of glioma. NANOG expression is regulated by p53, a cancer suppressor, which when lost, led to NANOG overexpression. The latter overexpression in glioma led to increase in its growth. NANOG was also found to be regulator of focal adhesion kinase (FAK), a matricellular protein responsible for motility of tumor cells in metastases. NANOG overexpression is indirectly induced by the use of temozolomide through c-MET activation. Despite its therapeutic resistance effect, resveratrol is a promising therapy for limiting NANOG expression. The findings in the present review argues for NANOG utilization as a therapeutic target for glioma.

Biomed Rev 2020; 31: 41-47

Keywords: transcription factor, NANOG, p53, glioma, cancer stem cells, malignancy biomarker, resveratrol

Received 17 November 2020, revised 2 December 2020, accepted 5 December 2020

*Correspondence to: Dr Novi Silvia Hardiany, Department Biochemistry and Molecular Biology, Faculty of Medicine, Universitas Indonesia, Salemba Raya No. 6, Jakarta Pusat, Indonesia

Phone:+62213910734 Email: novi.silvia@ui.ac.id 


\section{INTRODUCTION}

Glioma is a major cause of primary brain tumor with a worldwide prevalence of 6.0 per 100,000 persons. These tumors are difficult to treat due to the vulnerable brain parenchyma in which the tumor is located. Because of high risk of treatment, the disease is a leading cause of morbidity and mortality (1). As with other types of tumors, researchers have identified a population of cells in glioma that act as the renewing cells for tumor conferring immortality to the tumor, the cancer stem cells. In glioma, they are specifically called glioma stem cells with marker of CD133+ (2). These cells create self-renewability, proliferation, and differentiation potential to the tumor, and in addition confers therapeutic resistance, specifically to temozolomide which is the standard chemotherapy for glioblastoma (GBM), a glioma of grade IV malignancy based on the WHO classification. These cancer stem cells may hold the key to the metastasizing and proliferative ability of tumor (3).

Tumorigenesis originates from cancer stem cells that have molecular signatures similar to the embryonic stem cells. $N A$ $N O G$ is a gene coding for a transcription factor containing a DNA binding domain; it is one of the key gene for maintenance of stem cell capabilities that renders a cell to longevity. The name NANOG derived from Tir nan Og, a mythical land of youth of the Celtics. Along with SOX2 and OCT-4, NANOG comprises a key regulatory gene of embryonic stem cells. Studies has confirmed the crucial role of NANOG in maintaining pluripotency and self-renewability of embryonic stem cells. Within the cancer tissue, population of cancer stem cells are found that would be absent in the normal tissue. Similarities between embryonic stem cells and cancer stem cells include intensive growth and high expression of telomerase that imparts the immortality to the cells. NANOG is almost not expressed in normal somatic cells, except for a minute expression from fibroblast. Its expression has been characterized in several types of tumor such as brain, breast, cervix, colon, gastric, head and neck tumor. It has also been linked to resistance to therapies and poor prognosis (4).

While NANOG effects has been characterized in several tumors, its role in the tumorigenesis of glioma is minimally understood. In addition, characterizing the molecular events in cancer stem cells remains challenging because population of cancer stem cells in glioma are heterogeneous with varying molecular features (5). In the present review the role of NANOG in glioma malignancy and its potential as therapeutic target will be elaborated.

\section{CHARACTERISTIC OF STEMNESS FACTORS IN GLIOMA}

NANOG is highly expressed in pluripotent cells such as the embryonic stem cells and germ cells, while almost absent in the mature somatic cells with the exception of fibroblast. From both neural stem cells and glioma, the stem cells genes' expression of SOX2, OCT4, and NANOG have been detected. Holmberg et al compared expression of NANOG, SOX2, and OCT4 self-renewal factors in neural stem cells and glioma. NANOG and OCT4 was significantly overexpressed in the GBM. In addition, they are often found to be co-expressed with SOX2 in the high-grade glioma. The key difference between neural stem cells and glioma is that in neural stem cells, neural proteins are not expressed, while in glioma both neural proteins and the stem cell proteins are both expressed. This supports the notion that the expression of stem cell genes is needed for the progression of glioma in terms of growth and malignancy (3).

Cancer stem cells express similar factors with embryonic stem cells. In glioma SOX2 and OLIG2 have been identified to be expressed in glioma cells and the stem cells of human brain in which both are crucial in maintaining self-renewal of stem cells in the CNS (3). Their role in tumorigenesis is shown by the effect of SOX2 loss that limits the self-renewal capacity of glioma and in turn reduces their tumorigenesis effect. This was demonstrated in a study by Gangemi et al (6) in which they knockdown SOX2 gene in GBM using a retroviral vector carrying microRNA (miRNA) that was specifically engineered for silencing SOX2. GBM used in the study was obtained from glioma samples obtained in resection surgery. Their study showed, despite various mutation, GBM with silenced SOX2 stops proliferating as shown by the decreased expression of $\mathrm{Ki}-67$ which is a proliferation marker.

In addition to SOX2, OCT4 is another transcription factor that holds a key role in regulating stem cells characteristics. OCT4 is not expressed in adult brain cells, but has been shown to be expressed in high-grade glioma along with its downstream effectors. Study by Shi et al (7) demonstrated that importance of OCT4 in malignancy of glioma, where they found high methylation of OCT4 in the lower grade glioma compared to high grade glioma. In the same study, they also performed demethylation of glioma using 5-Aza2 -deoxycytidine and found increased expression of OCT4 following demethylation treatment of glioma from two cells lines U87 and U215.

Niu et al (8) observed expression of NANOG in brain tumor stem cells isolated from U87 glioma cell line and found 
that mRNA ${ }^{\mathrm{NANOG}}$ and protein were highly expressed compared to the parental cells. This overexpression was highly correlated to expression of CD133 and with increasing glioma pathological grade. However, NANOG overexpression alone appear to be incapable of eliciting tumorigenesis. In mammary for instance, it cannot independently induce tumor, but when co-expressed with Wnt-1 tumorigenesis and metastasis of mammary resulted (9).

The mechanism of NANOG in tumorigenesis is not well understood because it has been found to behave unlike classical oncogene such as OCT4. OCT4 overexpression in mouse cause dysplasia and tumor-like growth in skin, but in contrast NANOG overexpression although can cause growth but does not induce dysplastic phenotype. Based on this observation, NANOG seems to function as protumorigenic molecule that requires cooperation with other molecule to exert its tumor inducing effect. This could be because stem cell properties regulation of cancer stem cells involved multiple interaction of gene expression. This form of regulation differs from that of embryonic stem cells, in which single gene expression reflects the stemness property of cell (5).

\section{NANOG SIGNALING REGULATION IN GLIOMA}

$N A N O G$ gene was first discovered by Chambers et al (10) and Matsui et al (11) in mouse embryonic stem cells, where it acts as regulator of repressors and activators of differentiation. It is located on chromosome 12 which contains a homeobox domain. In embryonic stem cells, expression of NANOG is regulated by TCF3 and 533 through negative regulation. These two proteins regulate $N A N O G$ by binding to its promoter. Another genes that regulate $N A N O G$ expression are $L I F$ and $B M P$, that acts through their downstream proteins STAT3 (4). LIF that is capable of maintaining undifferentiated state in embryonic stem cells, can induce phosphorylation of STAT3 that directly binds to a NANOG protein enhancer. Activated STAT has been found to regulate NANOG in several tumors such as hepatocarcinoma and breast cancer cells. In breast cancer cells NANOG was found to form complex with STAT3 that later regulate their common target gene $(4,12)$. Hedgehog $(\mathrm{HH})$, a regulator of self-renewal, regulate NANOG in brain tumors through its downstream effectors called GLI1 and GLI2. These two proteins bind to NANOG promoter and activate NANOG expression (13). HH-GLI signaling pathway activates NANOG and is required for the growth of GBM. It was demonstrated that this interaction creates a positive loop, in which NANOG is also needed for GLI expression as NANOG, along with OCT4 and SOX2, can decrease inhibition of HH-GLI pathway $(13,14)$.

\section{NANOG ROLE IN MALIGNANCY OF GLIOMA}

\section{Metastases}

In their study to elucidate the role of NANOG in breast tumorigenesis Lu et al (15) found that when co-expressed with Wnt-1 in mouse mammary gland, NANOG promotes migration and invasion of breast cancer cell, lung cancer and ovary cancer. In another study, co-expression of NANOG and OCT4 was found to promote epithelial to mesenchymal transformation (EMT) that is needed for tumor to migrate and invade other region. Epithelial to mesenchymal transformation mediated via NANOG interactions with its downstream molecules which leads to initiation of metastases. NANOG overexpression facilitates migration by promoting the formation of filopodia and lamellipodia. NANOG interacts with platelet-derived growth factor receptor- $\alpha$ (PDGFR- $\alpha$ ) to mediate the formation of invadopodia as a response to EMT (16). PDGFR- $\alpha$ has functions to stimulate cell proliferation and migration. This receptor was found to be amplified in oligodendroglial tumors of grade II and III (17). In another pathway through EMT, NANOG interacts with focal adhesion kinase (FAK). The latter is known to be involved in cell adhesion, motility, survival and proliferation. It is overexpressed in many types of tumor including glioma (17). Autophosphorylation of FAK in response to alteration of extracellular matrix, growth factor signaling and integrin clustering cause changes in the cytoskeletal organization that increases cellular motility, and consequently tumor invasion. While inhibitors of FAK phosphorylation may decrease cellular invasion. Proteomics analyses have shown that FAK expression increase in response to exogenous introduction of NANOG to cells. NANOG binds to the promoter of FAK and increases the promoter activity. Also in the FAK promoter is the binding site of $\mathrm{p} 53$, that functions to inhibit transcription of FAK both in-vitro and in-vivo (18). FAK and NANOG interacts in positive loop, as it phosphorylates NANOG in a dose-dependent manner. This interaction appears to be a direct one through binding of NANOG at the N-terminal of FAK (19).

\section{Self-renewal}

NANOG and HH is both known to be regulators of selfrenewal in cancer stem cells. NANOG role in self-renewal was demonstrated in the study by Zbinden et al (20) where GBM 
cancer stem cells with knockdown NANOG resulted in reduction in number of cells compared to glioma without knockdown NANOG in a single cell clonogenic assays over 2 week period. The reduction was as much as around $20-80 \%$ cell numbers. This shows NANOG is essential for clonogenic growth of the GBM stem cells (14). NANOG role in self-renewal of cancer stem cells is through insulin-like growth factor 1 receptor (IGF1R). There is decrease of IGF1R expression in cancer stem cells with $N A N O G$ gene that has been silenced. IGF1R pathway is well known to contribute in progression of several tumors including glioma through its downstream signaling that includes PI3K/AKT and MAPK/ERK1/2. In addition, MAPK is also potent inducer of factors involved in EMT in tumor (21).

\section{Apoptosis and cell cycle}

In GBM U87 cell line, percentage of cell undergoing apoptosis appear to be significantly increased when NANOG is knock downed. NANOG anti-apoptotic pathway is regulated by AICAR-AMPK-p53 pathway. Jiang et al showed this by introduction of GSK621, which is an AMP-activated protein kinase (AMPK) activator, to glioma and saw an anti-tumor effect through activation of caspase-dependent apoptotic pathway in glioma cell line U87 $(22,23)$. AICAR is 5-aminoimidazole-4-carboxyamide ribonucleoside that activates
AMPK. Downstream to AMPK is p53, an apoptotic-inducing tumor suppressor (24). p53 and NANOG appear to regulate each other negatively. p53 suppression of NANOG in apoptosis occurs with translocation of $\mathrm{p} 53$. This translocation is suspected to be a response towards reactive oxygen species. p53 translocation to the nuclear is regulated by SIRT, a p53 deacetylase, that inhibits p53 activation and consequently apoptosis (25). Role of NANOG in cell cycle was shown by Chen et al (26) in observation of cells with knockdown NANOG. The authors showed that knocking down NANOG in mouse embryonic cells resulted in increase of the ratio of $\mathrm{G}_{\mathrm{o}} / \mathrm{G}_{1}$, cells meaning more cells are in the resting phase and decreases percentage of cells in $\mathrm{S}$ phase. As mentioned, p53 phosphorylation negatively affects NANOG expression and results in cell cycle arrest (26). The cell cycle arrest effect however occurs via p53 activation, as knockdown of p53 does not produce the same effect even when AICAR is activated.

The schematic summary of NANOG signal regulation in glioma is depicted in Figure 1.

\section{IMPLICATION OF NANOG OVEREXPRESSION IN PROGNOSIS AND THERAPIES}

Some malignancy features such as resistance to therapy has been associated with the presence of the cancer stem cells.

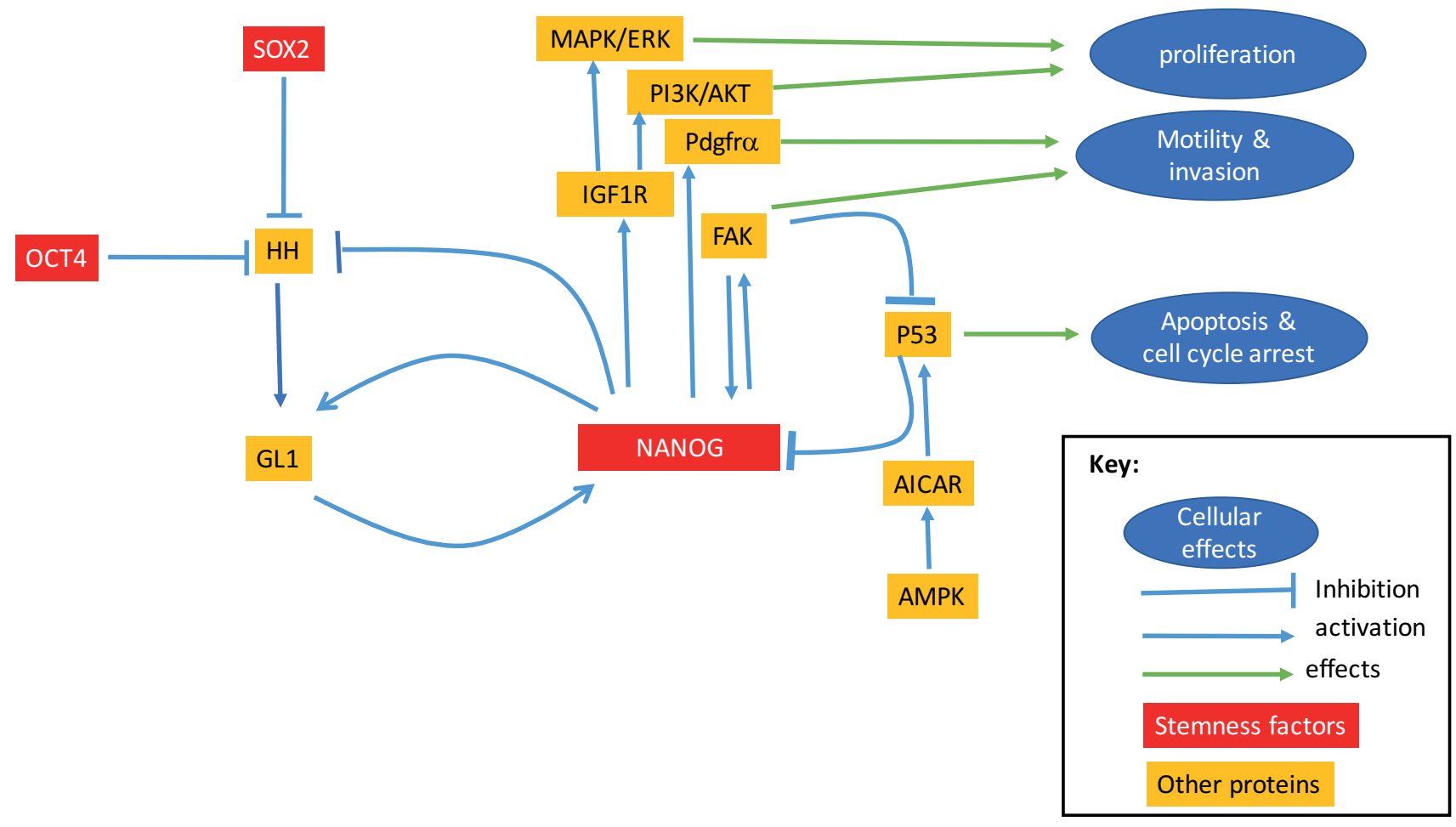

Figure 1. Schematic summary of NANOG signal regulation and pathways to produce cellular malignant behavior in glioma. 
Hence. eradication of cancer stem cells may be the most promising cancer therapy (5). GBM as one of the most malignant cancer in adults with average deaths of 12 months after diagnosis have very limited treatment option. Being highly invasive, it is not resolved with surgical resection, as residual tumor will remain invasive (27).

NANOG has been excessively associated with poorer prognosis in different type of cancer. In colorectal cancer, it was found that overexpression of NANOG was strongly correlated with poor prognosis, and lymph node metastases. On cellular level, NANOG overexpression promote motility, proliferation and migration in colorectal cancer cells obtained from patients (28).

In a different study, correlation analysis has established the strong relationship between NANOG overexpression and liver metastases. The same effect is also seen in oral cancer, whereby high expression of NANOG was associated with progression to a higher stage and poorer prognosis (29).

GBM is shown to have resistance to radiotherapy and chemotherapy. It has been suggested that the resistance can be attributed to cancer stem cells due to its proficiency in DNA damage repair by phosphorylation of CHK1/CHK2. Inhibitor of these DNA repair checkpoints kinase was effective in reversing the radio resistance in the glioma stem cells thus eliminating the cells that is a potential source of tumor cell repopulation after radiotherapy (30). GBM is infamous for its resistance to temozolomide. This resistance has been investigated to be affected by Adducin 3 (ADD3) an actin binding-cytoskeleton protein. It has been identified as responsible for causing chemoresistance in several types of cancer such as osteosarcoma (31). AAD3 us a tetrameric protein that induces actin binding resulting in plasma membrane folding. Upregulation of ADD3 was seen in glioma stem cells that were positive with markers such as CD133, OCT3/4, and NANOG has been associated with GBM resistance to temozolomide, giving the foundation for further investigation into ADD3 potential role as biomarker and target of temozolomide-resistant glioma (32).

Study on the effect of current therapies to stem cell markers should also be pursued as they may lead to further tumorigenesis instead of eradication. This type of response from a cancer therapy is exhibited by GBM exposed to anti-EGFR therapy that resulted in overexpression c-MET. c-MET displays selfrenewability and pluripotency. Downstream, c-MET signals proceeds to activation of stem cells factors such as OCT4 and NANOG. This result indicates EGFR as a promising agent in controlling tumorigenic propagation by arresting NANOG activity (33).

Another promising therapy that suppresses NANOG activity in cancer stem cells is resveratrol as demonstrated in study by Sato et al (34) that there was significant reduction of self-renewal capacity of patient-derived glioma stem cells. Treatment with resveratrol activates p53/p21 pathway, and in addition MG132 which is a proteasome inhibitor that degrades NANOG (34).

\section{CONCLUSION}

Glioma is a solid tumor of the brain that is difficult to treat. Within a tumor, there a population of cells that render the tumor its growing and metastasizing capacity, which are called the cancer stem cells. These cells have molecular signatures that are not found in the normal-differentiated cells. One of these signatures is the overexpression of NANOG, a stem cell marker responsible for self-renewal, pluripotency and metastasizing capacity of tumor cells. Together with HH, NANOG regulates self-renewal in tumor cells. In another pathway, its interaction with p53 inhibit apoptosis, while its interaction with PDGFR- $\alpha$ and FAK promote motility to tumor cells to facilitate metastases. Not just promotion of malignancy, NANOG implicated radiotherapy and chemotherapy resistance. With these multiple aspects of NANOG role in tumor progression, it is a promising target for glioma eradication that should be further explored.

\section{CONFLICT OF INTEREST}

Authors declare no conflict of interest exists.

\section{REFERENCES}

1. de Robles P, Fiest KM, Frolkis AD, Pringsheim T, Atta C, St. Germaine-Smith C, et al. The worldwide incidence and prevalence of primary brain tumors: a systematic review and meta-analysis. Neuro-Oncol 2015; 17(6): 776-83. DOI: 10.1093/neuonc/nou283.

2. Bien-Möller S, Balz E, Herzog S, Plantera L, Vogelgesang $\mathrm{S}$, Weitmann $\mathrm{K}$, et al. Association of glioblastoma multiforme stem cell characteristics, differentiation, and microglia marker genes with patient survival. Stem Cells 2018; 2018: 1-19. DOI: 10.1155/2018/9628289.

3. Holmberg J, He X, Peredo I, Orrego A, Hesselager G, Ericsson $\mathrm{C}$, et al. Activation of neural and pluripotent stem cell signatures correlates with increased malignancy in human glioma. PLoS ONE 2011; 6(3): e18454. DOI: 10.1371/journal.pone.0018454. 
4. Gong S, Li Q, Jeter CR, Fan Q, Tang DG, Liu B. Regulation of NANOG in cancer cells: nanog regulation in cancer cells. Mol Carcinog. 2015; 54(9): 679-87. DOI: 10.1002/ mc. 22340

5. Balbous A, Cortes U, Guilloteau K, Villalva C, Flamant S, Gaillard A, et al. A mesenchymal glioma stem cell profile is related to clinical outcome. Oncogenesis 2014; 3(3): e91-e91. DOI: 10.1038/oncsis.2014.5

6. Gangemi RMR, Griffero F, Marubbi D, Perera M, Capra MC, Malatesta $\mathrm{P}$, et al. SOX2 silencing in glioblastoma tumor-initiating cells causes stop of proliferation and loss of tumorigenicity. Stem Cells 2009; 27(1): 40-8. DOI: 10.1634/stemcells.2008-0493

7. Shi J, Shi W, Ni L, Xu X, Su X, Xia L, et al. OCT4 is epigenetically regulated by DNA hypomethylation of promoter and exon in primary gliomas. Oncol Rep 2013; 30(1): 201-6. DOI: 10.3892/or.2013.2456

8. Niu C-S. Expression of NANOG in human gliomas and its relationship with undifferentiated glioma cells. Oncol Rep 2011; 26(3): 593-601. DOI: 10.3892/or.2011.1308

9. Jeter CR, Yang T, Wang J, Chao H-P, Tang DG. Concise review: NANOG in cancer stem cells and tumor development: An update and outstanding questions: Nanog and tumor development. Stem Cells 2015 ; 33(8): 2381-90. DOI: $10.1002 /$ stem.2007

10. Mitsui K, Tokuzawa Y, Itoh H, Segawa K, Murakami M, Takahashi $\mathrm{K}$, et al. The homeoprotein nanog is required for maintenance of pluripotency in mouse epiblast and ES cells. Cell 2003; 113(5): 631-42. DOI: 10.1016/s00928674(03)00393-3

11. Chambers I, Colby D, Robertson M, Nichols J, Lee S, Tweedie $\mathrm{S}$, et al. Functional expression cloning of $\mathrm{Na}-$ nog, a pluripotency sustaining factor in embryonic stem cells. Cell 2003; 113(5): 643-55. DOI: 10.1016/s00928674(03)00392-1

12. Pan G, Thomson JA. Nanog and transcriptional networks in embryonic stem cell pluripotency. Cell Res 2007; 17(1): 42-9. DOI: 10.1038/sj.cr.7310125

13. Brandner S. NANOG, Gli, and p53: a new network of stemness in development and cancer. EMBO J 2010; 29(15): 2475-6. DOI: 10.1038/emboj.2010.162

14. Zbinden M, Duquet A, Lorente-Trigos A, Ngwabyt S-N, Borges I, et al. NANOG regulates glioma stem cells and is essential in vivo acting in a cross-functional network with GLI1 and p53. EMBO J 2010; 29(15):2659-2674. DOI: $10.1038 / \mathrm{emboj} .2010 .137$
15. Lu X, Mazur SJ, Lin T, Appella E, Xu Y. The pluripotency factor nanog promotes breast cancer tumorigenesis and metastasis. Oncogene 2014; 33(20): 2655-2664. DOI: 10.1038/onc.2013.209

16. Johnson DS, Mortazavi A, Myers RM, Wold B. Genomewide mapping of in vivo protein-DNA interactions. Science 2007; 316(5830): 1497-1502. DOI: 10.1126/ science.1141319

17. Gladson CL, Prayson RA, Liu WM. The pathobiology of glioma tumors. Аппи Rev Pathol Mech Dis 2010; 5(1): 33-50. DOI: 10.1146/annurev-pathol-121808-102109

18. Golubovskaya VM, Cance WG. FAK and p53 protein interactions. Anticancer Agents Med Chem 2011; 11(7): 617-619. DOI: $10.2174 / 187152011796817619$

19. Ho B, Olson G, Figel S, Gelman I, Cance WG, Golubovskaya VM. NANOG increases focal adhesion kinase (FAK) promoter activity and expression and directly binds to FAK protein to be phosphorylated. J Biol Chem 2012; 287(22): 18656-18673. DOI: 10.1074/jbc.M111.322883

20. Gawlik-Rzemienniewska N, Bednarek I. The role of NANOG transcriptional factor in the development of malignant phenotype of cancer cells. Cancer Biol Ther 2016; 17(1):1-10. DOI: 10.1080/15384047.2015.1121348.

21. Shi Z-M, Wang X-F, Qian X, Tao T, Wang L, Chen Q-D, et al. MiRNA-181b suppresses IGF-1R and functions as a tumor suppressor gene in gliomas. RNA N Y N 2013; 19(4): 552-60. DOI: 10.1261/rna.035972.112

22. Jiang H, Liu W, Zhan S-K, Pan Y-X, Bian L-G, Sun B, et al. GSK621 targets glioma cells via activating AMPactivated protein kinase signalings. PloS One 2016; 11(8): e0161017. DOI: 10.1371/journal.pone.0161017

23. Chen T, Du J, Lu G. Cell growth arrest and apoptosis induced by Oct 4 or NANOG knockdown in mouse embryonic stem cells: a possible role of Trp53. Mol Biol Rep 2012; 39(2): 1855-1861. DOI: 10.1007/s11033-011-0928-6.

24. Fridman JS, Lowe SW. Control of apoptosis by p53. Oncogene 2003; 22(56): 9030-9040. DOI: 10.1038/ sj.onc. 1207116

25. Han M-K, Song E-K, Guo Y, Ou X, Mantel C, Broxmeyer HE. SIRT1 regulates apoptosis and NANOG expression in mouse embryonic stem cells by controlling p 53 subcellular localization. Cell Stem Cell 2008; 2(3): 241-251. DOI: 10.1016/j.stem.2008.01.002.

26. Chae H-D, Lee M-R, Broxmeyer HE. 5-Aminoimidazole4-carboxyamide ribonucleoside induces G1/S arrest and nanog downregulation via p53 and enhances erythroid 
differentiation. Stem Cells 2012: 30(2): 140-149. DOI: 10.1002/stem.778

27. Seymour T, Nowak A, Kakulas F. Targeting aggressive cancer stem cells in glioblastoma. Front Oncol 2015; 5:159. DOI: 10.3389/fonc.2015.00159

28. Meng H-M, Zheng P, Wang X-Y, Liu C, Sui H-M, Wu S-J, et al. Over-expression of Nanog predicts tumor progression and poor prognosis in colorectal cancer. Cancer Biol Ther 2010; 9(4): 295-302. DOI: 10.4161/cbt.9.4.10666

29. Xu F, Dai C, Zhang R, Zhao Y, Peng S, Jia C. NANOG: A potential biomarker for liver metastasis of colorectal cancer. Dig Dis Sci 2012; 57(9): 2340-2346. DOI: 10.1007/ s10620-012-2182-8.

30. Bao S, Wu Q, McLendon RE, Hao Y, Shi Q, Hjelmeland $\mathrm{AB}$, et al. Glioma stem cells promote radioresistance by preferential activation of the DNA damage response. Nature 2006; 444(7120): 756-760. DOI: 10.1038/nature 05236 .
31. Walters DK, Steinmann P, Langsam B, Schmutz S, Born W, Fuchs B. Identification of potential chemoresistance genes in osteosarcoma. Anticancer Res 2008; 7. PMID: 18507006

32. Poon MW, Zhuang JTF, Wong STS, Sun S, Zhang XQ, Leung GKK. Co-expression of cytoskeletal protein adducin 3 and CD133 in neurospheres and a temozolomideresistant subclone of glioblastoma. Anticancer Res 2015; 9. PMID: 26637861

33. Jun HJ, Bronson RT, Charest A. Inhibition of EGFR induces a c-MET-driven stem cell population in glioblastoma: MET is required for stemness in glioblastoma. Stem Cells 2014; 32(2): 338-348. DOI: 10.1002/stem.1554

34. Sato A, Okada M, Shibuya K, Watanabe E, Seino S, Suzuki K, et al. Resveratrol promotes proteasome-dependent degradation of NANOG via p53 activation and induces differentiation of glioma stem cells. Stem Cell Res 2013; 11(1): 601-610. DOI: 10.1016/j.scr.2013.04.004 